\title{
Characteristics of Boiling Heat Transfer on Hydrophobic Surface
}

\author{
Anton Surtaev ${ }^{1,2}{ }^{*}$, Vladimir Serdyukov ${ }^{1,2}$, and Alexey Safonov ${ }^{1,2}$ \\ ${ }^{1}$ Kutateladze Institute of Thermophysics, 630090 Novosibirsk, Russia \\ ${ }^{2}$ Novosibirsk State University, 630090 Novosibirsk, Russia
}

\begin{abstract}
The paper presents the results of an experimental study of the effect of hydrophobic fluoropolymer coating on the multiscale characteristics of heat transfer at water boiling. New experimental data on dynamics of vapor bubble growth and detachment, evolution of contact line, nucleation site density, heat transfer coefficient were obtained using high-speed imaging techniques, including infrared thermography and video recording from the bottom side of transparent ITO heater. It was shown, that the using of hydrophobic fluoropolymer coating leads to heat transfer enhancement, to decrease of the superheat temperature at the onset of boiling, to increase of the active nucleation site density and to significant change in the dynamics of growth and departure of vapor bubbles and the evolution of the triple contact line.
\end{abstract}

\section{Introduction}

It is well-known that the properties of heat exchange surfaces have a significant influence on dynamics of phase transition, in particular on liquid boiling [1]. Wettability is one of the key parameters, since it displays interaction dynamics between the liquid, vapor and heating solid wall. Wetting properties of the surface have shown significant influence on bubble dynamics, heat transfer and critical heat fluxes (CHF) at liquid boiling [2]. Nowadays a lot of attention is paid to the investigation on the influence of hydrophobic coatings on various aspects of liquid boiling. Such interest is associated with the need to reduce the superheating at the onset of nucleate boiling, as well as with the possibility of using such coatings for fouling inhibition during boiling of aqueous solutions [3], which is very important task for improvement the efficiency of heat exchange equipment. In addition, some authors have shown that the usage of hydrophobic coatings makes it possible to significantly improve the heat transfer intensity at nucleate boiling [4-7]. However there are some studies, which indicate negative effect of the increase of contact angle on the heat transfer coefficient at boiling [8].

However, despite the fact that the wetting properties have a significant effect on the local characteristics of the process and heat transfer intensity at boiling, now there are no reliable theoretical models for describing the observed phenomena. Insufficient understanding of the physics of the process is connected, among other things, with a limited

* Corresponding author: surtaev@itp.nsc.ru 
array of experimental information. Recently, the development of modern experimental methods, including high-speed infrared thermography, video recording, laser interferometry, etc. for visualization of the process from the bottom side of a transparent substrate with a thin film heater, allows us to obtain fundamentally new information on nucleation dynamics and heat transfer characteristics at liquid boiling [2]. Despite the prospects of the above-described high-speed measurement techniques, there are practically no studies in literature that would allow one to study the effect of nanocoatings with different wetting properties on the local and integral characteristics of heat transfer at liquid boiling using these methods. This would make it possible to achieve a significant progress in understanding the fundamental laws governing the influence of wetting properties on the most important parameters characterizing the boiling process.

In this paper the experimental results on the effect of hydrophobic coatings on the internal characteristics of the process (density of nucleation sites, dynamics of vapor bubble growth and departure) and heat transfer at water boiling are presented. Data were obtained with the use of simultaneous high-speed video and infrared recordings with high temporal and spatial resolution, and special construction of on a transparent heater.

\section{Experimental techniques}

\subsection{Pool boiling experiments}

The detailed description of the experimental setup, heating element and measurement techniques can be founded in [9]. Milli-Q water after the degassing procedure was used as the working liquid. In the experiments $1-\mu \mathrm{m}$-thick films of indium-tin oxide (ITO), vacuum deposited onto 400- $\mu \mathrm{m}$-thick sapphire substrates, were used as the heaters. Such construction of the heating element was recently widely used for pool boiling experiments and showed possibility to measure non-stationary temperature field on the ITO film surface by infrared camera and visually record vapor bubbles dynamics on the sapphire substrate by high-speed video camera. Fabricated samples had the heating area of $20 \times 20 \mathrm{~mm}^{2}$.

To visualize boiling process with high spatial and temporal resolutions digital video camera Vision Research Phantom v.7.0 with frame rate up to 1000 FPS and maximum spatial resolution of $24 \mu \mathrm{m} /$ pix was used. Visualization was performed from the bottom side of the transparent heater. As it will be shown below, this format of visualization allowed us to study in detail the dynamics of triple contact line in the base of vapor bubbles and nucleation site density at boiling in the range of low heat fluxes. To study the shape of the growing and departing bubbles the video recording from the side of the heater also was performed. In the experiments high-speed thermographic (IR) camera FLIR Titanium was used to measure the non-stationary temperature field of the heating surface. As configured for this study, the thermographic camera had a frame rate of 1000 FPS and maximum resolution of $150 \mu \mathrm{m} /$ pix.

\subsection{Surface modification}

The hydrophobic fluoropolymer films were deposited on the sapphire substrate via HotWire Chemical Vapor Deposition technique (HWCVD). This method is one of the promising for producing fluoropolymer coatings suitable for phase transitions applications, such as condensation and boiling since it allows controlling the morphology (i.e. roughness) of a deposited film by changing the deposition process parameters. In addition, the method allows to use various substrate materials. In this work fluoropolymer films from $\mathrm{C}_{3} \mathrm{~F}_{6} \mathrm{O}$ precursor gas, activated by $\mathrm{NiCr}$ wire catalyst mesh were deposited on the substrate 
at varying deposition process parameters such as activator temperature, the precursor gas pressure and precursor gas flow rate. The thickness of the films in different modes was varied by changing the deposition time. Detailed description of HWCVD method can be found in [10]. After the synthesis, the physico-chemical properties of each sample were analyzed using a complex of methods, including nanoprofiometry, electronic spectroscopy and microscopy (EDX, SEM), and KRUSS setup for measuring free surface energy and contact angle. The thickness of fabricated coating was $310 \mathrm{~nm}$. According to analysis of the water droplet shape on the substrate (Fig. 1), the fluoropolymer coating has contact angle of $120^{\circ}$, which is much higher than the value measured on the surface of pure glass and sapphire $\left(\theta \sim 60^{\circ}\right)$.

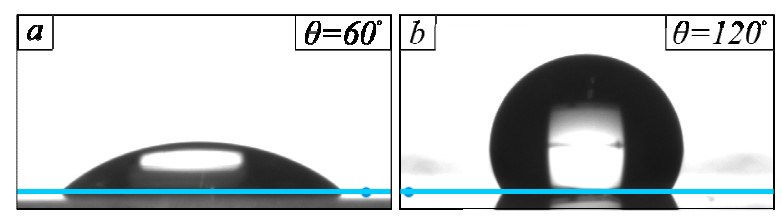

Fig. 1. Contact angle measurement with water droplet $(V=5 \mu \mathrm{l}): a)$ on the pure sapphire substrate; $b$ ) on the fluoropolymer coated surface.

It should be noted, that the measurement of contact angle value also was performed after the boiling experiments and showed the same results. The analysis of coating morphology showed that the roughness value $R_{a}$ does not exceed $20 \mathrm{~nm}$, which is higher than $R_{a}$ value for the surface of polished sapphire, but it is significantly smaller than the characteristic value of the critical vapor nucleus for observed superheating of liquid at water boiling.

\section{Results and Discussion}

To construct boiling curves, experimental data on the heating surface temperature obtained by IR thermography were averaged over the recording time and surface area at different heat flux densities. Corresponding boiling curves for water on the heating surfaces with hydrophobic fluoropolymer coating and without it are presented in Fig. 2a. Analysis of data shows that the heat transfer intensity at water boiling on the hydrophobic surface are more than 3 times higher than on the uncoated sapphire surface at heat flux density up to $q=45$ $\mathrm{kW} / \mathrm{m}^{2}$. Similar heat transfer enhancement was observed in [5] at water pool boiling on slightly hydrophobic surface $\left(\theta \approx 108^{\circ}\right)$. Moreover, in comparison with uncoated surface, the activation of first nucleation sites at boiling on hydrophobic coating occurs already at low surface superheating $\left(\Delta T \approx 1.5^{\circ} \mathrm{C}\right)$. Authors of $[5,11]$ also observed the formation of first vapor bubbles at low superheating $\left(\Delta T \approx 3-4{ }^{\circ} \mathrm{C}\right)$ at boiling on slightly hydrophobic coatings. For comparison, for float glass surface without coating this value was about $19{ }^{\circ} \mathrm{C}$ [5].

To determine the possible mechanisms of the influence of hydrophobic coatings on heat transfer, following local boiling characteristics were studied: growth rates of vapor bubbles and triple contact line area, bubble departure diameters, nucleation site density (NSD), etc. The $N S D$ value was measured using high-speed video recording for various heat fluxes (Fig. 2b). Analysis shows that the NSD for water boiling on the surface with hydrophobic coating is higher for the given heat fluxes than for boiling on the uncoated sapphire surface. Since the roughness of the used hydrophobic coating is significantly less than the critical radius of heterogeneous nucleation at boiling, the increase of the number of active nucleation sites on the hydrophobic surface in comparison with smooth heater is primarily due to the change of wetting properties of the heating surface rather than its morphology. 
An increase in the nucleation site density at boiling on smooth hydrophobic coatings was also noted by authors of $[4,5]$.
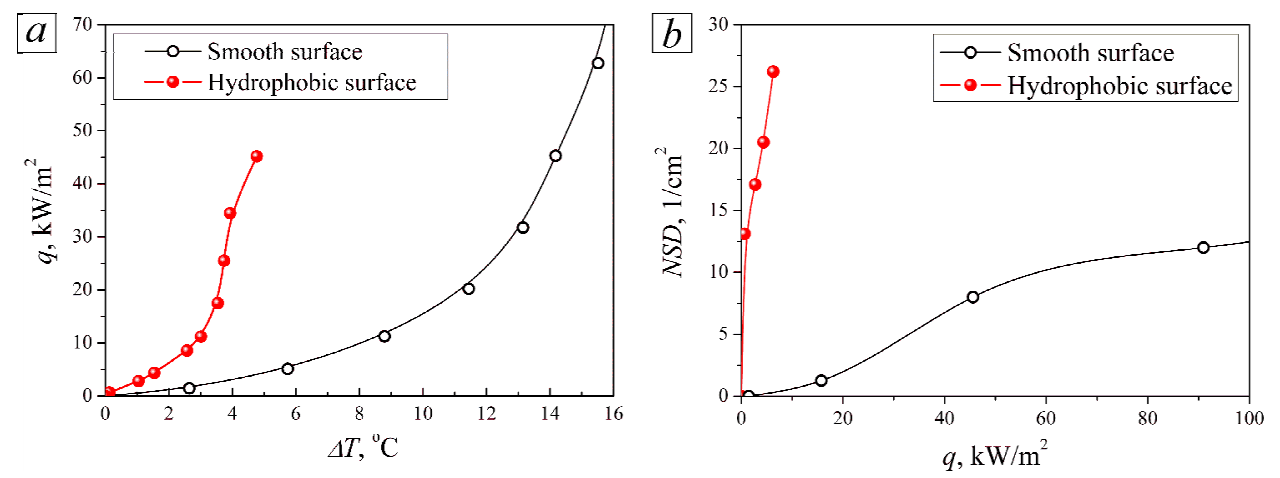

Fig. 2. a) Boiling curves for saturated water on the pure sapphire surface and surface with hydrophobic fluoropolymer coating; $b$ ) Dependence of nucleation site density on heat flux at water pool boiling on surface with different wettability.

The decrease of the superheat temperature at the onset of boiling on hydrophobic surface follows from the analysis of the thermodynamic potential change (Gibbs energy) for metastable liquid when a vapor nucleus appears on a solid wall at different wetting properties. Moreover authors of [5] suggested that an increase in the NSD value at boiling on hydrophobic surface may be related to the existence of vapor-gas bubbles on a heating surface, which can act as ready nucleation sites. At boiling on a hydrophobic surface in contrast to uncoated surface such bubbles are stable and have tend to settle on the surface, which is associated with the presence of an interface with chemical heterogeneities [5].

Fig. 3 shows frames of high-speed video recording of the vapor bubbles dynamics at boiling on the surface with fluoropolymer hydrophobic coating at $q=8.5 \mathrm{~kW} / \mathrm{m}^{2}$. In these frames, we can clearly see the outer diameter of the bubbles base and the region of triple contact line for separated bubbles.
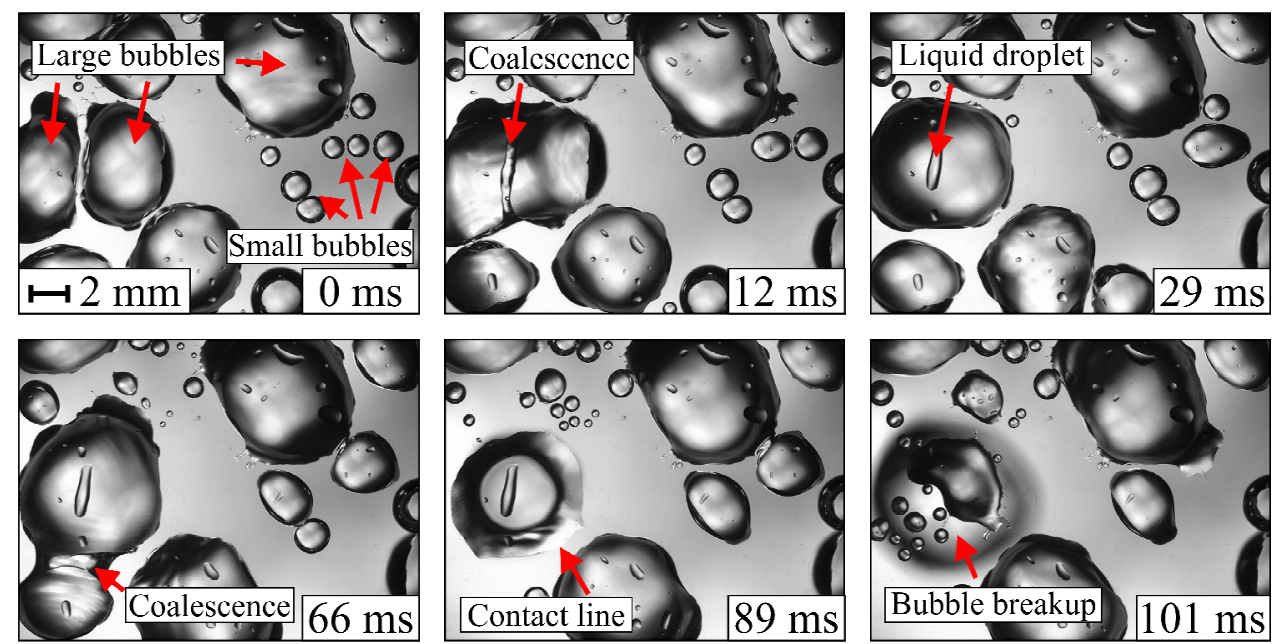

Fig. 3. Frames of high-speed macro visualization of vapor bubbles dynamics from the bottom side of the heater at water boiling on the sapphire surface with hydrophobic coating $\left(q=8.5 \mathrm{~kW} / \mathrm{m}^{2}\right)$.

From the figure it can be seen that there are two different types of vapor bubbles on the surface: larger - «sessile» and smaller, for which an increase in the diameter of the bubble 
base and the area of the triple contact line is observed. In contrast to boiling on the uncoated surface, such bubbles don't have the area of intensive evaporation of the microlayer under the bubble. Fig. 4 shows the evolution of diameter of vapor bubble base and contact line area at boiling on hydrophobic surface and uncoated heater. The comparative analysis shows, that at boiling on hydrophobic coating the growth rates of vapor bubble and area bounded by the triple contact line are smaller, than at boiling on uncoated surface, while the bubble lifetime is significantly longer. It can also be seen that, unlike boiling on uncoated smooth surface, where the stage of vapor bubble departure is accompanied by contact line reduction, at boiling on hydrophobic surface contact line area monotonously increases along with bubble growth.

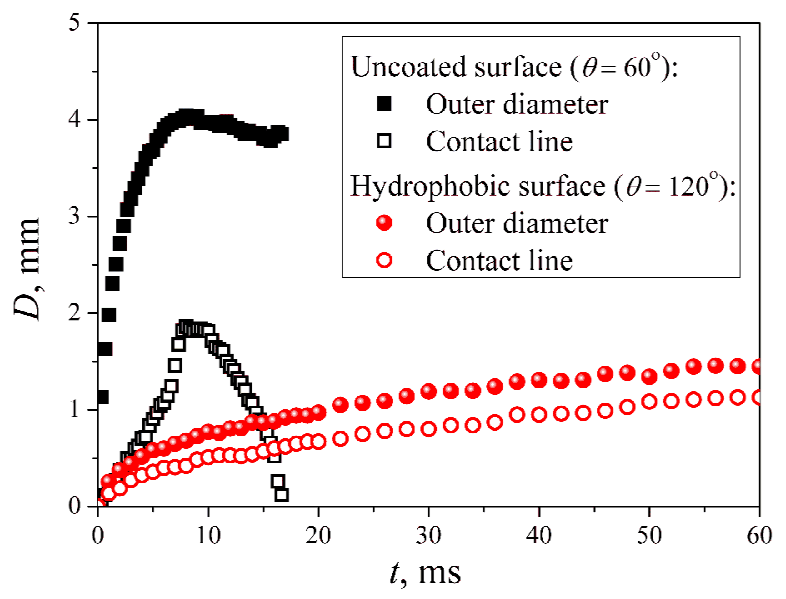

Fig. 4. Evolution of the outer diameter of a single bubble and the contact line at water boiling on the uncoated surface and surface with hydrophobic coating $\left(q=5 \mathrm{~kW} / \mathrm{m}^{2}\right)$.

Also, in contrast to case of uncoated surface, where the departure of vapor phase from the heating surface is accompanied by contact line reduction, at boiling on hydrophobic surface another picture is observed. At the stage of growth, before the achievement of size corresponding to the departure diameter of bubbles on uncoated surface, bubbles coalesce with neighbor nucleation sites with an increase in the area of contact line. It also should be noted, that the coalescence of bubbles into large vapor conglomerates occurs at the some distance from the heating surface, as a result of which liquid separating them may remain after the coalescence under vapor phase in the form of droplets. After the departure of large vapor bubbles from the surface in this place numerous vapor bubbles remain. This bubbles start to grow, merge and form new large bubble.

The analysis of video recording from the side of heating surface (Fig. 5) shows that the large, sessile bubbles have "bell" shape with stretched interface, which corresponds to apparent contact angle between wall and vapor more than $90^{\circ}$. Such bubbles can exist on the hydrophobic surface for a long time, which is associated with an increase in the surface tension force at the boundary of three phases.

This force, as it was shown in [11] opposes the shrinking of the bubble base, which leads to the fact that the emission frequency of bubbles on hydrophobic surface is about 1 $\mathrm{Hz}$, which is significantly lower than at boiling on uncoated surface. At the departure of large bubbles from the heater they start to pull up and the breaking of vapor "neck", which connects the upper part of the bubble and its base, occurs. After the departure of the bubble main volume, some vapor still remains on the surface as nuclei of the next bubble's initiation. The similar behavior of large vapor bubbles at boiling on hydrophobic surface was observed in $[7,11]$. 

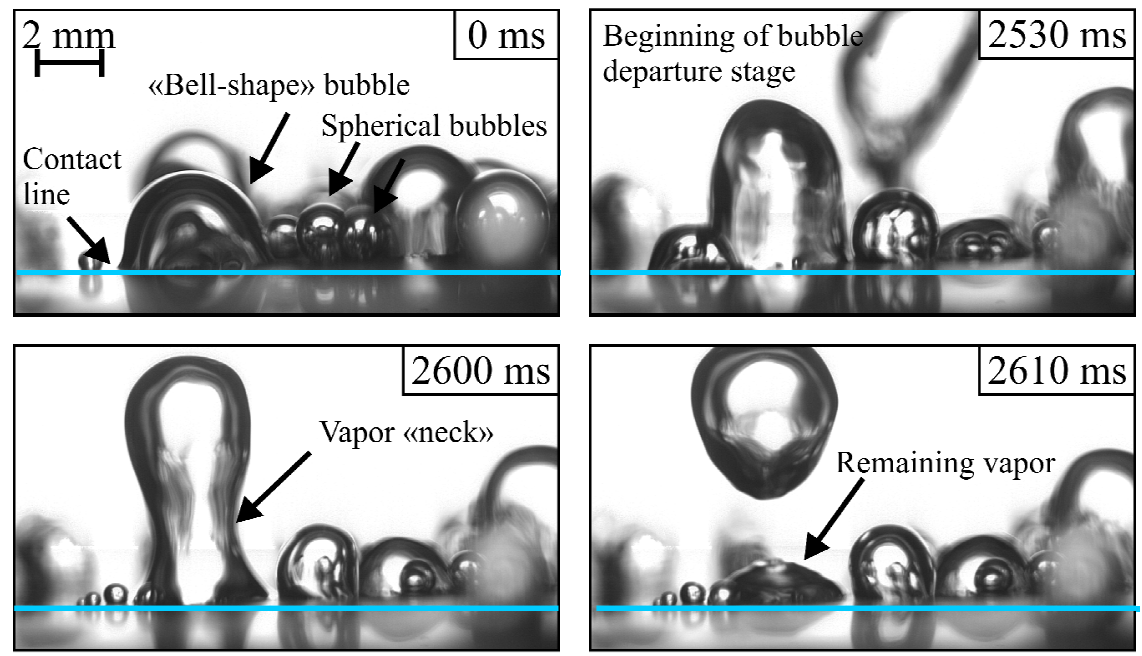

Fig. 5. Frames of high-speed visualization from the side of the heater at water boiling on the hydrophobic fluoropolymer coating $\left(q=11.2 \mathrm{~kW} / \mathrm{m}^{2}\right)$.

\section{Conclusions}

The effect of fluoropolymer hydrophobic nanocoating on the multiscale characteristics of saturated water boiling at atmospheric pressure was studied using high-speed video visualization and IR thermography and comparison with data obtained on uncoated sapphire substrate was made. It was shown, that:

- Significant heat transfer enhancement (up to 3 times) is observed at boiling on hydrophobic coating in the region of low heat fluxes in comparison with data obtained on uncoated sapphire surface [9];

- With the deterioration of surface wetting characteristics, the nucleation site density value increases significantly and the superheat temperature at the onset of boiling significantly decreases at given heat fluxes;

- It has been shown that the growth rate of bubbles at boiling on hydrophobic surface is significantly lower in comparison with uncoated surface at the same heat fluxes. Investigation of the bubbles evolution showed that at water boiling on fluoropolymer nanocoating there is not area of microlayer evaporation.

The reported study was funded by the Russian Science Foundation (Project № 18-79-00078).

\section{References}

1. A. S. Surtaev, V. S. Serdyukov, A. N. Pavlenko, Nanotech. in Russia 11, 696 (2016).

2. D. Attinger et al., MRS Energy Sustainability 1, E4 (2014).

3. L. Hui, M. Liu et al., Chemical Eng. \& Technology 38(3), 416 (2015).

4. A. R. Betz et al., Int. J. of Heat and Mass Transfer 57(2), 733 (2013).

5. B. Bourdon et al., Adv. in Colloid and Interface Science 221, 34 (2015).

6. J. S Kim et al., Int. Journal of Heat and Mass Transfer 118, 802 (2018).

7. H. J. Jo et al., Int. Journal of Heat and Mass Transfer 54(25-26), 5643 (2011).

8. E. Teodori et al., Applied Thermal Engineering 115, 1424 (2017). 
9. A. S. Surtaev et al., Int. J. of Heat and Mass Transfer 124, 297 (2018).

10. A. I. Safonov et al., Thin Solid Films 653, 165 (2018).

11. Y. Nam et al., Journal of Heat Transfer 131(12), 121004 (2009). 